Www.jmscr.igmpublication.org

Index Copernicus Value: 79.54

ISSN (e)-2347-176x ISSN (p) 2455-0450

crossref DOI: https://dx.doi.org/10.18535/jmscr/v7i4.79

Journal Of Medical Science And Clinical Research

IGM Publication

An Official Publication of IGM Publication

\title{
Efficacy of Photodynamic Therapy and Diode laser therapy as an adjunct to Scaling and Root Planing in the treatment of Chronic Periodontitis - A Clinical \& Microbiologic Short Term Study
}

\section{Authors \\ Dr Savita $S^{1}$, Dr Sunita R. Pal ${ }^{2^{*}}$, Dr Anuroopa $\mathbf{P}^{3}$}

${ }^{1}$ Principal, Professor \& Head of Department, Department of Periodontology, Rajarajeswari Dental College and Hospital, Bangalore-560074

${ }^{2}$ Post graduate, Department of Periodontology, Rajarajeswari Dental College and Hospital, Bangalore-560074

${ }^{3}$ Reader, Department of Periodontology, Rajarajeswari Dental College and Hospital, Bangalore-560074

*Corresponding Author

\section{Dr Sunita R. Pal}

Post graduate, Dept of Periodontology, Rajarajeswari Dental College and Hospital, Bangalore-560074, India Tel: +91 9611654096; Email: sunitarpal1989@ gmail.com

\begin{abstract}
Introduction: Chronic periodontitis is treated routinely by scaling \& root planing. An innovative therapy represented by the irradiation of periodontal pockets with laser \& photodynamic therapy can be considered as a promising novel approach for eradicating pathogenic bacteria in periodontitis.

Aim and Objectives: To compare the efficacy of Diode laser \& Photodynamic therapy with SRP and SRP alone in chronic periodontitis patient.

Methodology: A total of 11 patients aged between 30 - 50 years diagnosed as chronic generalised periodontitis were selected for the study \& grouped as:

Group 1 (10 sites were treated with scaling and root planing alone).

Group 2 (10 sites were treated with scaling and root planing followed by diode laser therapy),

Group 3 (10 sites were treated with scaling \& root planing followed by methylene blue mediated photodynamic therapy)

The clinical parameters were evaluated at baseline, $1^{\text {st }}$ week $\& 3^{\text {rd }}$ week interval after therapy, Subgingival plaque samples were collected using sterilized curette and total bacterial count were evaluated.

Results: Significant reduction in clinical \& microbial parameters was seen in group 2 and group 3 i.e., reduction in mean CFUs, However, greater statistically significant reduction was observed in group 3.

Conclusion: Photodynamic therapy is a valuable treatment modality adjunctive to conventional scaling and root planing in comparison with diode laser therapy.

Keyword: Lasers, Root planing, Photosensitizer, Chronic periodontitis, Reactive oxygen species.
\end{abstract}

\section{Introduction}

Periodontal disease is initiated by pathogenic plaque biofilm and characterized bybacteria- induced inflammatory destruction of toothsupporting structures and alveolar bone ${ }^{1}$. Mechanical scaling and root debridement have 
shown to be an effective treatment approach for periodontal disease. However, the limitations of scaling and root debridement have also been shown in management of initially deep periodontal pockets and fraction involved lesions ${ }^{2}$. In recent years, various innovative adjunctive treatments have therefore been developed to improve the clinical effectiveness of scaling and root debridement.

A novel non-invasive approach for infection control, namely diode laser, has been receiving much attention in the treatment of oral diseases ${ }^{3}$. In addition to SRP the use of laser therapy, as shown by several studies, appears to improve and facilitate the healing of treated sites ${ }^{4}$. A diode laser with a wavelength of $810-980 \mathrm{~nm}$, may have considerable antibacterial potential by direct ablation, thermal denaturation, or other destruction of bacterial cells. The diode lasers may reduce bacteria with fewer thermal side effects.

Photodynamic therapy (PDT) has been introduced as a method of antimicrobial treatment that may kill periodontal bacteria with even fewer adverse effects. PDT is based on the principle that a photoactive substance, the photosensitizer, binds to the target cell and can be activated by a light of suitable wavelength. Following activation of the photosensitizer, singlet oxygen and other very reactive agents are produced that are extremely toxic to certain cells and bacteria. The photosensitizer is generally applied in the targeted area by topical application, aerosol delivery or interstitial injection. Clinical effects of these new treatment methods have been studied to a certain degree, but the results are rather heterogeneous.

Various studies have shown the use of laser associated to photosensitizer which is very efficient against bacteria, yeasts, viruses and parasites. Hence, the aim of the present prospective, controlled, clinical study is to evaluate clinically and microbiologically the effectiveness of the adjunctive use of photodynamic therapy with scaling and root planning versus lasers in non-surgical periodontal treatment in patients with chronic periodontitis.

\section{Materials \& Methods}

This clinical and microbiologic study was carried out in the Department of Periodontology, Rajarajeswari Dental College \& Hospital, Bangalore. The study protocol was reviewed and approved by the Institutional Review board of Rajarajeswari dental college \& hospital and Ethical clearance was obtained for the study. The nature of the study along with recall protocol was explained to the patients and a written consent was obtained before commencing the study.

A total of 11 patients aged between $30-50$ years diagnosed as chronic generalised periodontitis were selected in this study, out of which 30 sites are treated and grouped as follows: Group 1 (10 sites, treated with scaling and root planing), Group 2 (10 sites, treated with scaling and root planing followed by Doide laser therapy), and Group 3 (10 sites, treated with scaling and root planing followed by methylene blue mediated photodynamic therapy).

Inclusion criteria for the study were; systemically healthy patients, patient should have more than 20 teeth remaining, more than $30 \%$ of sites involved, presence of periodontal pocket in at least two teeth with a probing depth of $>5 \mathrm{~mm}$ ineach quadrant. Patients with history of systemic diseases affecting the periodontium, smokers and alcoholic patients, pregnant and lactating women, patients who have undergone periodontal treatment 6 months prior to the study were excluded from the study. A total of 90 samples from 30 sites were collected at baseline first week and third week after treatment. Microbiological analyses of the collected samples were performed.

\section{Collection of data}

After screening and selecting the subject for study, the clinical examinations were done pre and postoperatively at first and third week as follows. Plaque Index (Sillness and Loe) were recorded at baseline, 1st and 3rd week to observe the oral hygiene and motivation of the patients. Probing pocket depth (PPD) and clinical attachment level (CAL) measured from CEJ to base of the pocket 
were taken at baseline, first week and third week visit.(Fig 1)

\section{Plaque sampling for microbial analysis}

After removal of supra-gingival calculus and plaque, the area was isolated and dried with saliva evacuators\& cotton rolls, then collection of subgingival plaque samples were done using universal curettes (Hu-Friedy, USA.) from each selected site (Fig 1/2). Samples were collected at baseline, 1 st week and at $3^{\text {rd }}$ week postoperatively. The samples were placed in sterile vials containing $0.5 \mathrm{ml}$ of reduced transport fluid (RTF) and sent to the laboratory for microbial analysis(Fig 3 / 4). The mean colony forming units (CFUs/ml) of bacteria were counted by growing the colonies on sheep blood agar plates. The counts were done at the end of 2nd day after inoculation of the samples on the culture plates.

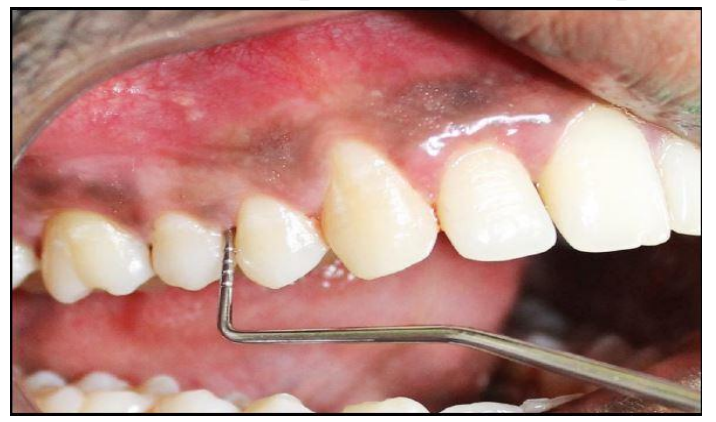

Fig 1: Pre- Operative Probing

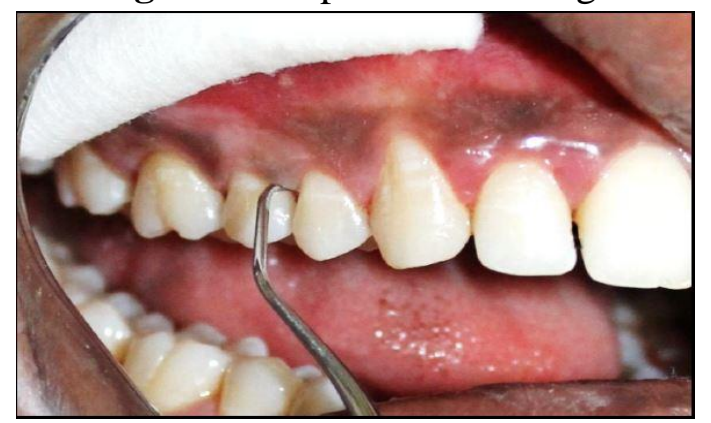

Fig 2: Dental Plaque Collection Using Curette

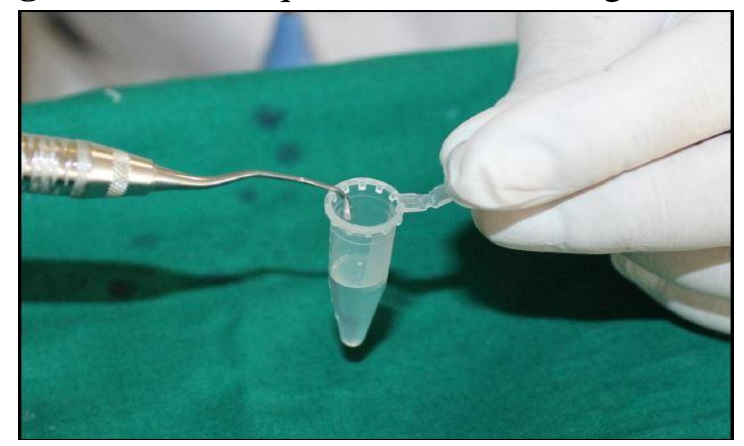

Fig 3: Transferring the Subgingival Plaque Sample in Storage Medium

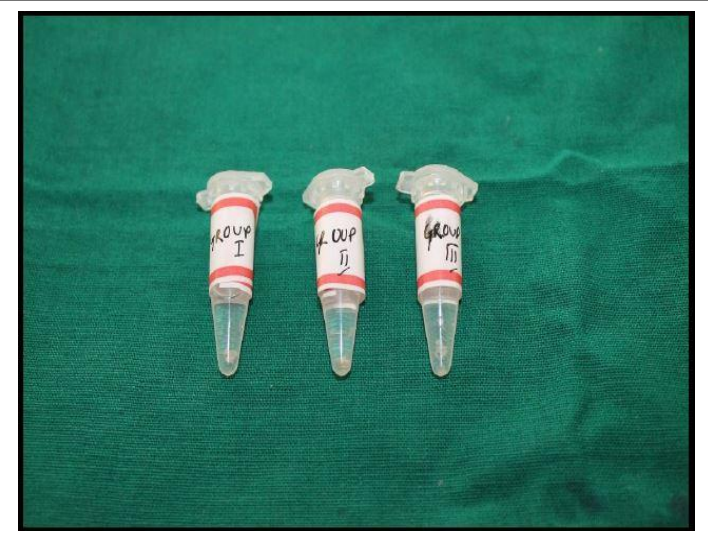

Fig 4: Collected Sample

The patients of the first group received initial preparation. It consists of mechanical instrumentation of the root surface, in order to make it biologically compatible with the healing of periodontal tissues by scaling and root planing procedure. The second group of patients received the same therapy did in the first group, but with the addition of laser therapy through a $940 \mathrm{~nm}$ diode and Group three received scaling and root planing along with photodynamic therapy.

Procedure for photodynamic therapy (PDT): After a preliminary oral rinse for 30 seconds, the periodontal pockets of selected teeth were filled with the $0.3 \%$ methylene blue solution from the bottom of the pocket using a blunt needle(Fig $5 / 6)$. After 3 minutes of action, the photosensitizer was rinsed with saline solution and the pocket was exposed to diode laser (wavelength940nm at $0.1 \mathrm{~W}$ power output, continuous mode) using a $320 \mu \mathrm{m}$ fiberoptic tip for 10 seconds in each site (Fig 7). The treatment was done in six sites per tooth totalling 1 minute of treatment per tooth.

The procedure was done using standard laser safety protocol including protective eye wear for the operator, patient and the assistant. The patients were then instructed for routine brushing and rinsing with water. The patients were informed about the complications like feeling of pressure, pain or irritation in the area and were advised to have soft diet. Only if the irritation became intolerable the patients were advised to report back to the department. 


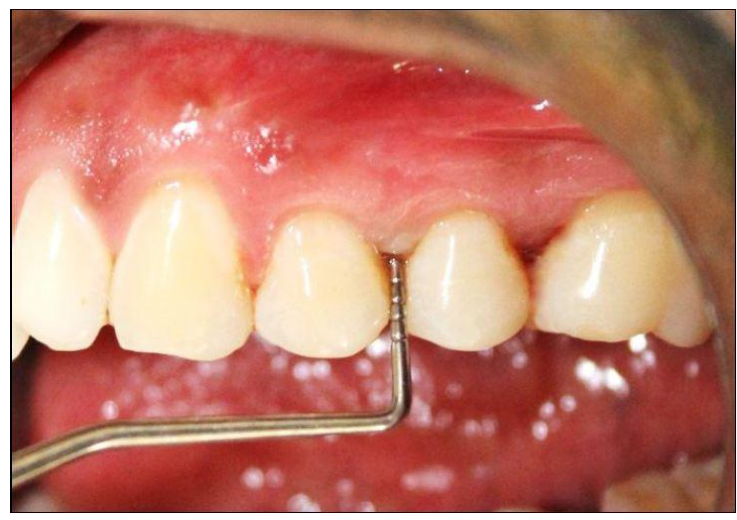

Fig 5: Pre-Operative Probing Depth

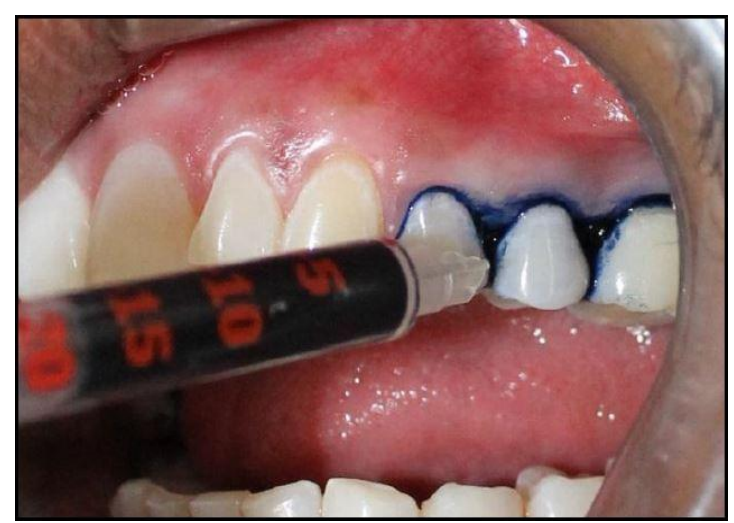

Fig 6: Application of Photosensitizer (Methylene Blue Dye)

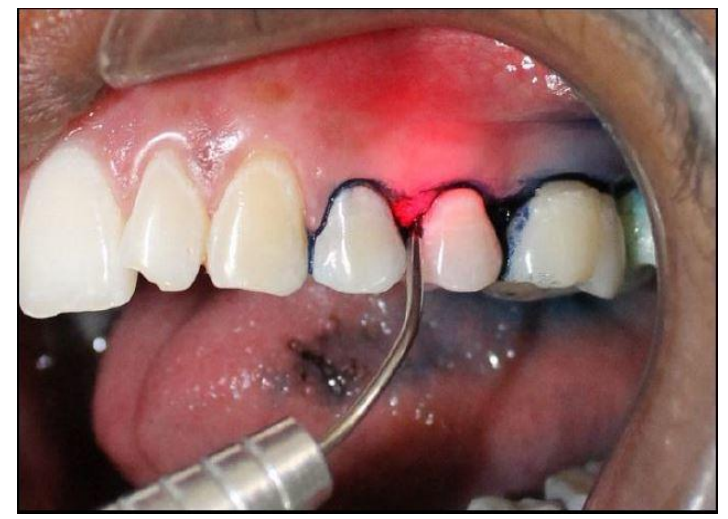

Fig 7: Irradiation with Laser

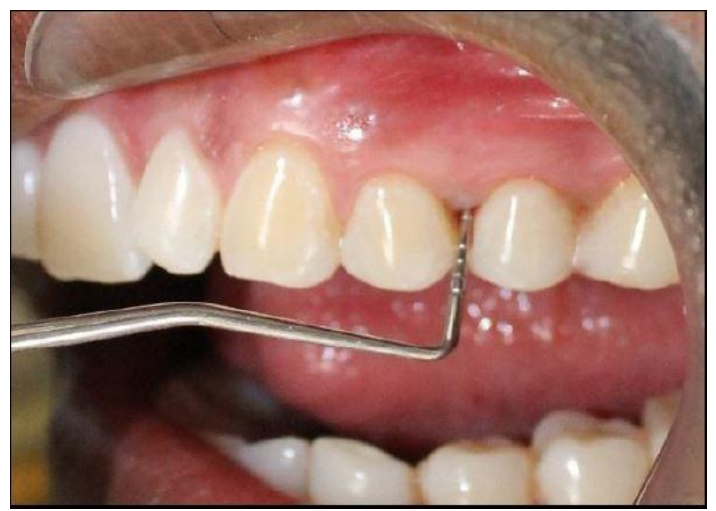

Fig 8: Post-Operative Probing Depth
All patients were recalled for professional prophylaxis and assessment of the clinical parameters 1 st and $3^{\text {rd }}$ week after the treatment (Fig 8).

\section{Statistical Analysis}

The data collected were entered in Microsoft Excel sheet and statistical analyses were performed using the Statistical Package for the Social Science (SPSS version 10.5) software. Mean age, PI, PPD and CAL were calculated per group. One-way analyses of variance were used to test the difference between groups. Post hoc Tukey test was used to find out the difference of mean values between two groups. In this test, $P<0.05$ was taken to be statistically significant. For colony forming units, a nonparametric version of ANNOVA, Kruskal-Wallis test is used.

\section{Results}

The study comprised of 11 subjects diagnosed as chronic generalised periodontitis within the age range of 30-50 years were included in the study. At baseline all the three study groups did not show any statistically significant differences in terms of clinical parameters (Graph 1). The test result demonstrated that, there was a statistically significant difference for mean plaque index score between the three groups (at $\mathrm{P}=0.002$ ), the multiple comparison test indicated that statistically significant difference were seen between group 1 and group 2 (at $\mathrm{P}=0.04$ ), and group 1 and group 3( at $\mathrm{P}=0.002$ ), however group 2 and group 3 did not differ statistically (Table 1).The periodontal pocket depth and clinical attachment level did not show any difference between the 3 groups at one week time interval.

At third week, the periodontal pocket and the clinical attachment loss along with plaque index all showed statistically significant difference with group 1 showing highest score in all 3 parameters, there was statistical difference between group 1 and group 2, and group 1 and group 3 (Table 2, 3/ 
Graph 2). In CFU group, at baseline there is no statistically significant difference in all the three groups. At one week time interval, there is statistically significant difference between the 3 groups, main statistically significant difference is there between group 2 and group 3, where group 3 is showing least mean colony forming units compared to group 1 and group 2. But at third week, there is significant difference between all the 3 groups, where group 3 is showing the least CFU followed by group 2 and group 1 (Graph 3).

Table 1

\begin{tabular}{|c|c|c|c|c|c|c|c|c|c|c|}
\hline \multicolumn{8}{|c|}{ Comparison of mean Plaque Index scores between different time intervals in each study group using Repeated } \\
measures of ANOVA test followed by Bonferroni's post hoc Analysis
\end{tabular}

Table 2

\begin{tabular}{|c|c|c|c|c|c|c|c|c|c|c|}
\hline \multicolumn{8}{|c|}{ Comparison of mean Pocket depth (in mm) between different time intervals in each study group using Repeated } \\
measures of ANOVA test followed by Bonferroni's post hoc Analysis
\end{tabular}

Table 3

\begin{tabular}{|c|c|c|c|c|c|c|c|c|c|c|}
\hline \multicolumn{11}{|c|}{$\begin{array}{l}\text { Comparison of mean CAL (in mm) between different time intervals in each study group using Repeated measures } \\
\text { of ANOVA test followed by Bonferroni's post hoc Analysis }\end{array}$} \\
\hline \multirow[b]{2}{*}{ Time } & \multicolumn{2}{|c|}{ Baseline } & \multicolumn{2}{|c|}{1 Week } & \multicolumn{2}{|c|}{3 Weeks } & \multirow[b]{2}{*}{ P-Value } & \multicolumn{3}{|c|}{ Bonferroni's Post hoc Analysis } \\
\hline & Mean & $\mathrm{SD}$ & Mean & $\mathrm{SD}$ & Mean & $\mathrm{SD}$ & & T1 Vs T2 & T1 Vs T3 & T2 Vs T3 \\
\hline Group 1 & 5.23 & 1.36 & 5.01 & 1.49 & 4.95 & 1.49 & $0.02^{*}$ & 0.11 & $0.04^{*}$ & $0.002^{*}$ \\
\hline Group 2 & 4.98 & 1.44 & 4.46 & 1.66 & 4.14 & 1.43 & $0.004^{*}$ & 0.13 & $0.006^{*}$ & $0.03^{*}$ \\
\hline Group 3 & 4.76 & 1.42 & 4.00 & 1.21 & 3.06 & 1.30 & $<0.001^{*}$ & $0.002^{*}$ & $<0.001^{*}$ & $<0.001^{*}$ \\
\hline
\end{tabular}

\section{Graph 1}

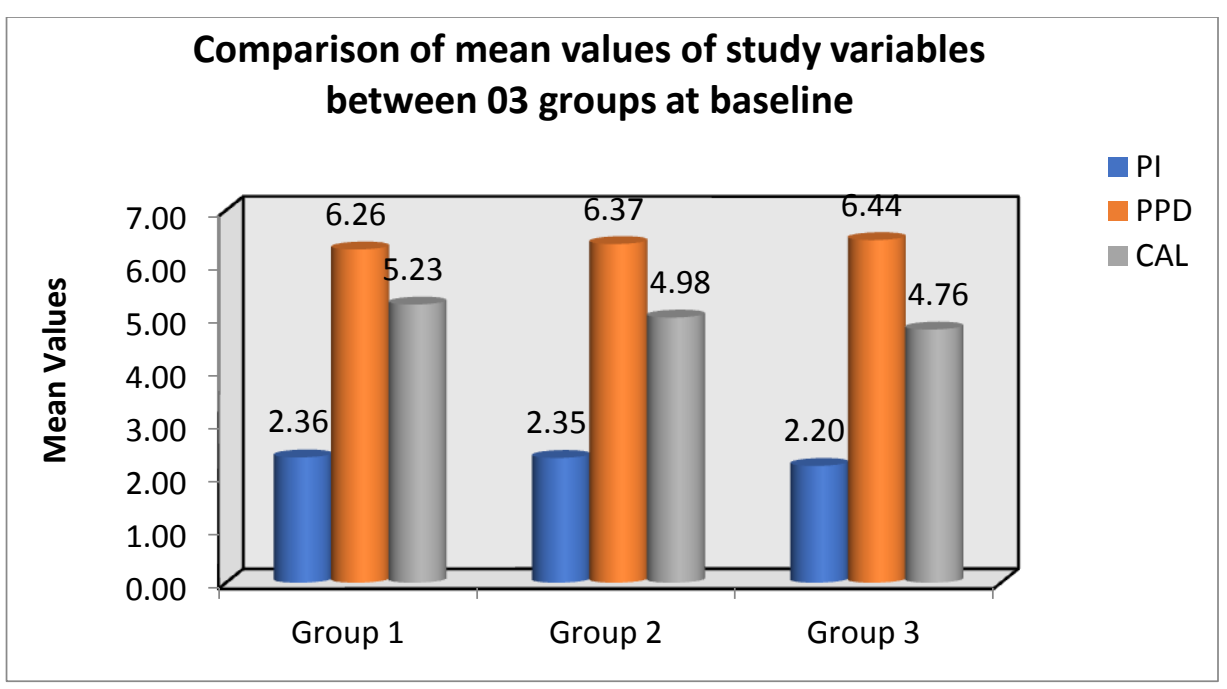


Graph 2:

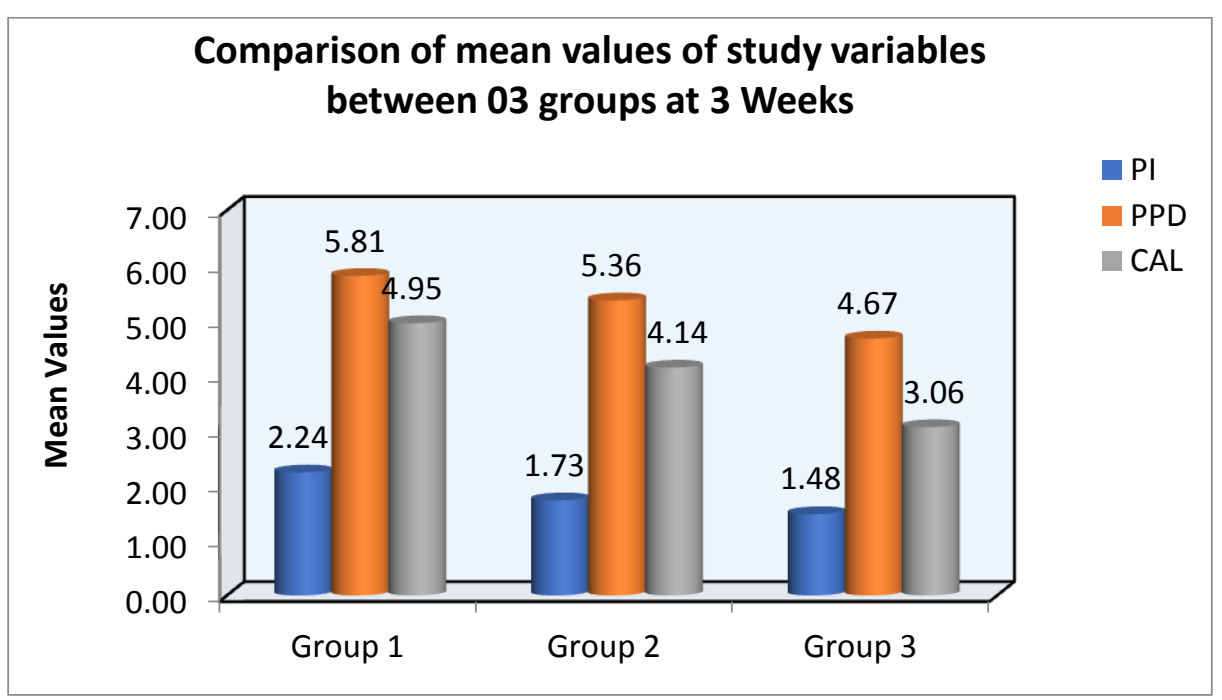

\section{Graph 3}

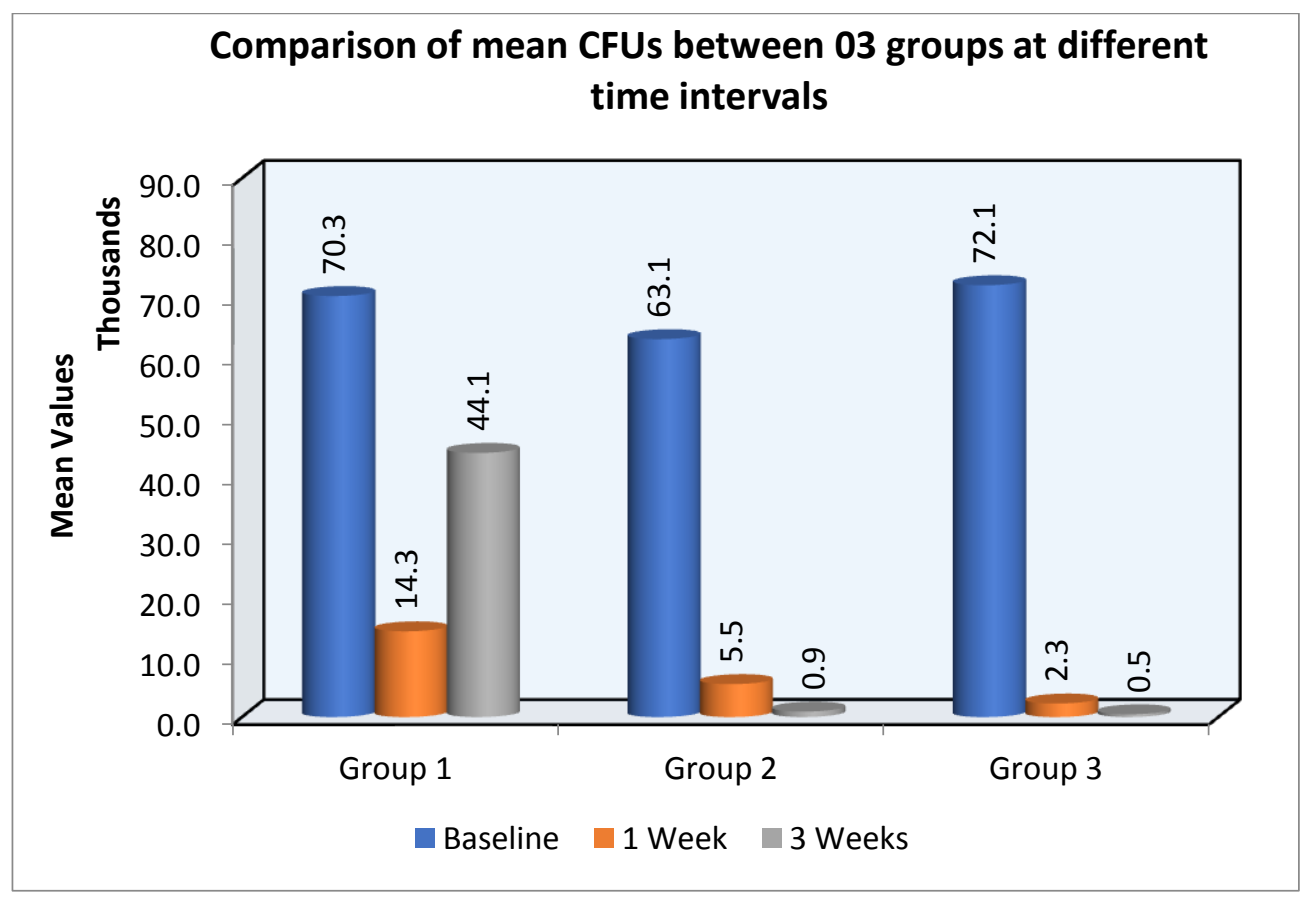

\section{Discussion}

The primary aim of periodontal therapy is the removal of supra and subgingival bacterial deposits by mechanical debridement consisting of scaling and root-planing (SRP). The complete removal of bacteria and their toxins from periodontal pockets is not always achieved with conventional mechanical treatment, hence it is supplemented with antibiotics ${ }^{5}$.The disadvantages of chemotherapy are possible bacterial resistance and side effects after systemic administration. Photodynamic therapy avoids these drawbacks by combining irradiation with the soft laser with the photosensitized dye.Methylene blue was chosen as a photosensitizer in this study, as it has been shown to be potentially one of the safest photosensitizers for the treatment of periodontal disease. It has a complementary benefit on sites with deep periodontal pockets, furcations and root concavity ${ }^{6}$.

Considered the best clinical results, the laser diode can be routinely associated with traditional nonsurgical mechanical therapy (SRP) in the treatment of periodontal pockets of patients with moderate to severe chronic periodontitis. In the present study patients with chronic periodontitis 
and the adjunctive use of antimicrobial photodynamic therapy to scaling and root planing may result, on a short-term basis (up to $3^{\text {rd }}$ week), in (i) comparable reductions in Plaque Index (PI) score when compared with scaling and root planing alone(ii) comparable higher probing pocket depth reductions and clinical attachment level gains when compared with scaling and root planing alone.(iii) comparable reductions in bacterial count when compared with scaling and root planning alone ${ }^{7}$.

The uses of Photodynamic therapy as an adjunctive therapy for periodontal disease have been shown to be bactericidal and could possibly target pathogens more effectively.

The combination of laser therapy with conventional procedures allows, in fact, to obtain a more effective decontamination of the pocket, in addition to this the recolonization by bacteria is slower than the sites treated only with scaling and root planing.Moritz et al. and Angelov et al. showed that diode laser therapy can significantly improve periodontal pocket healing and periodontal indices ${ }^{8}$.

Our results are in accordance with study done by Andersen et al. (2007) who showed that a combination of SRP and PDT (diode laser $670 \mathrm{~nm}$ and methylene blue as photosensitizer) in patients affected by moderate to advance periodontitis resulted in significant improvements in clinical outcomes. Braun et al. (2008) evaluated the effect of adjunctive antimicrobial photodynamic therapy (methylene blue + diode laser) in chronic periodontitis using a split-mouth design. The authors reported that the adjunctive use of PDT has a positive effect on treatment outcomes. Similar results were reported by Sreedhar et al. (2016), as they evaluated comparison between PDT \& Diode laser therapy in aggressive periodontitis patients and concluded that PDT showed significant improvement with regards to all clinical parameter and reduction in bacterial load.

\section{Conclusions}

A greater improvement in clinical \& microbiological parameters have been observed in this study with the utilization of lasers along with methylene-blue dye in photodynamic therapy when used as an adjunct to SRP, the mean microbial levels showed marked reduction in bacterial counts in the sites which received Photodynamic Therapy (PDT).

\section{References}

1. Saurabh H, Dipika K et al. The effect of indocyanine green-mediated photodynamic therapy as an adjunct to scaling and root planing in the treatment of chronic periodontitis: A comparative split-mouth randomized clinical trial. IJDR 2016; 27 : 609-617

2. Suryakanth M, S.Harinath Reddy et al. Clinical effects of photodynamic and lowlevel laser therapies as an adjunct to scaling and root planing of chronic periodontitis: A split-mouth randomized controlled clinical trial.IJDR 2016; 27: 121-126

3. Andreas Moritz, Ulrich $\mathrm{S}$ et al. Treatment of periodontal pockets with a diode laser.Lasers in Surgery and Medicine 1998; 22(5):302 - 311.

4. Antonio $\mathrm{C}$, Michele $\mathrm{M}$ et al. Effectiveness of a diode laser in addition to non-surgical periodontal therapy: study of intervention.2015 Jan-Mar; 6(1): 15-20

5. Charles $M$ et al. Clinical significance of non-surgical periodontal therapy: An evidence-based perspective of scaling and root planing.Journal Of Clinical Periodontology 2002; 2(Suppl. 2):6-16

6. Ancely F, Lecitia $\mathrm{F}$ et al. Methylene blue photodynamic therapy induces selective and massive cell death in human breast cancer cells.BMC Cancer (2017) 17:194

7. Betsy J, Presanthila J et al.Is Antimicrobial Photodynamic Therapy Effective as an Adjunct to Scaling and Root Planing in 
Patients with Chronic Periodontitis? A Systematic Review.Biomolecules. 2017 Dec; 7(4): 79.

8. Mohammadreza $\mathrm{T}$, Rojin $\mathrm{T}$ et al. Microbiological Efficacy of Photodynamic Therapy as an Adjunct to Non-surgical Periodontal Treatment: A Clinical Trial.J Lasers Med Sci 2016 Spring;7(2):126-130

9. Cohen RE, Ammons W (1996) Lasers in periodontics. J Periodontol 67: 826-830.

10. Akira Aoki, Katia $M$ et al. Lasers in nonsurgical periodontal therapy. Periodontology 2000; Vol. 36, 2004, 5997. 